BULLETIN (New Series) OF THE

AMERICAN MATHEMATICAL SOCIETY

Volume 45, Number 2, April 2008, Pages 229-245

S 0273-0979(08)01199-3

Article electronically published on February 12, 2008

\title{
REGULARITY PROPERTIES IN THE CLASSIFICATION PROGRAM FOR SEPARABLE AMENABLE C*-ALGEBRAS
}

\author{
GEORGE A. ELLIOTT AND ANDREW S. TOMS
}

\begin{abstract}
We report on recent progress in the program to classify separable amenable $\mathrm{C}^{*}$-algebras. Our emphasis is on the newly apparent role of regularity properties such as finite decomposition rank, strict comparison of positive elements, and $\mathcal{Z}$-stability, and on the importance of the Cuntz semigroup. We include a brief history of the program's successes since 1989, a more detailed look at the Villadsen-type algebras which have so dramatically changed the landscape, and a collection of announcements on the structure and properties of the Cuntz semigroup.
\end{abstract}

\section{INTRODUCTION}

Rings of bounded operators on Hilbert space were first studied by Murray and von Neumann in the 1930s. These rings, later called von Neumann algebras, came to be viewed as a subcategory of a more general category, namely, $\mathrm{C}^{*}$-algebras. (The $\mathrm{C}^{*}$-algebra of compact operators appeared for perhaps the first time when von Neumann proved the uniqueness of the canonical commutation relations.) A $\mathrm{C}^{*}$-algebra is a Banach algebra $A$ with involution $x \mapsto x^{*}$ satisfying the $\mathrm{C}^{*}$-algebra identity:

$$
\left\|x x^{*}\right\|=\|x\|^{2}, \forall x \in A .
$$

Every $\mathrm{C}^{*}$-algebra is isometrically $*$-isomorphic to a norm-closed sub-*-algebra of the $*$-algebra of bounded linear operators on some Hilbert space, and so may still be viewed as a ring of operators on a Hilbert space.

In 1990, the first-named author initiated a program to classify amenable normseparable $\mathrm{C}^{*}$-algebras via $\mathrm{K}$-theoretic invariants. The graded and (pre-)ordered group $\mathrm{K}_{0} \oplus \mathrm{K}_{1}$ was suggested as a first approximation to the correct invariant, as it had already proved to be complete for both approximately finite-dimensional $(\mathrm{AF})$ algebras and approximately circle $(\mathrm{AT})$ algebras of real rank zero $([16],[18])$. It was quickly realised, however, that more sensitive invariants would be required if the algebras considered were not sufficiently rich in projections. The program was refined, and became concentrated on proving that Banach algebra K-theory and positive traces formed a complete invariant for simple separable amenable $\mathrm{C}^{*}$ algebras. Formulated as such, it enjoyed tremendous success throughout the 1990s and early 2000s.

Received by the editors April 20, 2007, and, in revised form, October 1, 2007.

2000 Mathematics Subject Classification. Primary 46L35; Secondary 46L80.

Key words and phrases. $\mathrm{C}^{*}$-algebras, classification.

This work was partially supported by the Natural Sciences and Engineering Research Council of Canada. 
Recent examples based on the pioneering work of Villadsen have shown that the classification program must be further revised. Two things are now apparent: the presence of a dichotomy among separable amenable $\mathrm{C}^{*}$-algebras dividing those algebras which are classifiable via K-theory and traces from those which will require finer invariants, and the possibility - the reality, in some cases - that this dichotomy is characterised by one of three potentially equivalent regularity properties for amenable $\mathrm{C}^{*}$-algebras. (Happily, the vast majority of our stock-in-trade simple separable amenable $\mathrm{C}^{*}$-algebras have one or more of these properties, including, for instance, those arising from directed graphs or minimal $\mathrm{C}^{*}$-dynamical systems.)

Our plan in this article is to give a brief account of the activity in the classification program over the past decade, with particular emphasis on the now apparent role of regularity properties. After reviewing the successes of the program so far, we will cover the work of Villadsen on rapid dimension growth AH algebras, the examples of Rørdam and the second-named author which have necessitated the present re-evaluation of the classification program, and some recent and sweeping classification results of Winter obtained in the presence of the aforementioned regularity properties. We will also discuss the possible consequences for the classification program of including the Cuntz semigroup as part of the invariant (as a refinement of the $\mathrm{K}_{0}$ and tracial invariants).

\section{Preliminaries}

We denote by $\mathcal{K}$ the $\mathrm{C}^{*}$-algebra of compact operators on a separable infinitedimensional Hilbert space $\mathcal{H}$. For a $\mathrm{C}^{*}$-algebra $A$, we let $\mathrm{M}_{n}(A)$ denote the algebra of $n \times n$ matrices with entries from $A$. The cone of positive elements of $A$ will be denoted by $A_{+}$.

2.1. The Elliott invariant and the original conjecture. The Elliott invariant of a $\mathrm{C}^{*}$-algebra $A$ is the 4 -tuple

$$
\operatorname{Ell}(A):=\left(\left(\mathrm{K}_{0} A, \mathrm{~K}_{0} A^{+}, \Sigma_{A}\right), \mathrm{K}_{1} A, \mathrm{~T}^{+} A, \rho_{A}\right),
$$

where the K-groups are the Banach algebra ones, $\mathrm{K}_{0} A^{+}$is the image of the Murrayvon Neumann semigroup $\mathrm{V}(A)$ under the Grothendieck map, $\Sigma_{A}$ is the subset of $\mathrm{K}_{0} A$ corresponding to projections in $A, \mathrm{~T}^{+} A$ is the space of positive tracial linear functionals on $A$, and $\rho_{A}$ is the natural pairing of $\mathrm{T}^{+} A$ and $\mathrm{K}_{0} A$ given by evaluating a trace at a $\mathrm{K}_{0}$-class. The reader is referred to Rørdam's monograph [50] for a detailed treatment of this invariant. In the case of a unital $\mathrm{C}^{*}$-algebra the invariant becomes

$$
\left(\left(\mathrm{K}_{0} A, \mathrm{~K}_{0} A^{+},\left[1_{A}\right]\right), \mathrm{K}_{1} A, \mathrm{~T} A, \rho_{A}\right),
$$

where $\left[1_{A}\right]$ is the $\mathrm{K}_{0}$-class of the unit, and $\mathrm{T} A$ is the (compact convex) space of tracial states. We will concentrate on unital $\mathrm{C}^{*}$-algebras in the sequel in order to limit technicalities.

The original statement of the classification conjecture for simple unital separable amenable $\mathrm{C}^{*}$-algebras read as follows:

Conjecture 2.1. Let $A$ and $B$ be simple unital separable amenable $C^{*}$-algebras, and suppose that there exists an isomorphism

$$
\phi: \operatorname{Ell}(A) \rightarrow \operatorname{Ell}(B) .
$$

It follows that there is a $*$-isomorphism $\Phi: A \rightarrow B$ which induces $\phi$. 
It will be convenient to have an abbreviation for the statement above. Let us call it (EC).

2.2. Amenability. We will take the following deep theorem, which combines results of Choi and Effros ([8]), Connes ([10]), Haagerup ([29]), and Kirchberg ([35]), to be our definition of amenability.

Theorem 2.2. $A C^{*}$-algebra $A$ is amenable if and only if it has the following property: for each finite subset $\mathcal{G}$ of $A$ and $\epsilon>0$ there are a finite-dimensional $C^{*}$-algebra $F$ and completely positive contractions $\phi, \psi$ such that the diagram

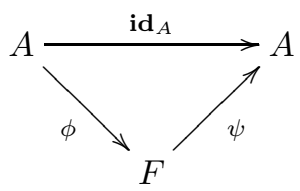

commutes up to $\epsilon$ on $\mathcal{G}$.

The property characterising amenability in Theorem 2.2 is known as the completely positive approximation property.

Why do we consider only separable and amenable $\mathrm{C}^{*}$-algebras in the classification program? It stands to reason that if one has no good classification of the weak closures of the GNS representations for a class of $\mathrm{C}^{*}$-algebras, then one can hardly expect to classify the $\mathrm{C}^{*}$-algebras themselves. These weak closures have separable predual if the $\mathrm{C}^{*}$-algebra is separable. Connes and Haagerup gave a classification of injective von Neumann algebras with separable predual (see [11] and [30]), while Choi and Effros established that a $\mathrm{C}^{*}$-algebra is amenable if and only if the weak closure in each GNS representation is injective ([9]). Separability and amenability are thus natural conditions which guarantee the existence of a good classification theory for the weak closures of all GNS representations of a given $\mathrm{C}^{*}$-algebra. The assumption of amenability (at least for a simple classification; cf. however [19]) has been shown to be necessary by Dădărlat ([15]).

The reader new to the classification program who desires a fuller introduction is referred to Rørdam's excellent monograph [50].

2.3. The Cuntz semigroup. One of the three regularity properties alluded to in the introduction is defined in terms of the Cuntz semigroup, an analogue for positive elements of the Murray-von Neumann semigroup $\mathrm{V}(A)$. It is known that this semigroup will be a vital part of any complete invariant for separable amenable $\mathrm{C}^{*}$-algebras ([56]). Given its importance, we present both its original definition, and a modern version which makes the connection with classical K-theory more transparent.

Definition 2.3 (Cuntz-Rørdam; see [13] and [54]). Let $\mathrm{M}_{\infty}(A)$ denote the algebraic limit of the direct system $\left(\mathrm{M}_{n}(A), \phi_{n}\right)$, where $\phi_{n}: \mathrm{M}_{n}(A) \rightarrow \mathrm{M}_{n+1}(A)$ is given by

$$
a \mapsto\left(\begin{array}{cc}
a & 0 \\
0 & 0
\end{array}\right)
$$

Let $\mathrm{M}_{\infty}(A)_{+}$(resp. $\left.\mathrm{M}_{n}(A)_{+}\right)$denote the positive elements in $\mathrm{M}_{\infty}(A)$ (resp. $\mathrm{M}_{n}(A)$ ). Given $a, b \in \mathrm{M}_{\infty}(A)_{+}$, we say that $a$ is Cuntz subequivalent to $b$ (written $a \precsim b)$ if there is a sequence $\left(v_{n}\right)_{n=1}^{\infty}$ of elements in some $\mathrm{M}_{k}(A)$ such that

$$
\left\|v_{n} b v_{n}^{*}-a\right\| \stackrel{n \rightarrow \infty}{\longrightarrow} 0
$$


We say that $a$ and $b$ are Cuntz equivalent (written $a \sim b$ ) if $a \precsim b$ and $b \precsim a$. This relation is an equivalence relation, and we write $\langle a\rangle$ for the equivalence class of $a$. The set

$$
\mathrm{W}(A):=\mathrm{M}_{\infty}(A)_{+} / \sim
$$

becomes a (positive) ordered Abelian semigroup when equipped with the operation

$$
\langle a\rangle+\langle b\rangle=\langle a \oplus b\rangle
$$

and the partial order

$$
\langle a\rangle \leq\langle b\rangle \Leftrightarrow a \precsim b .
$$

Definition 2.3 is slightly unnatural, as it fails to consider positive elements in $A \otimes \mathcal{K}$. This defect is the result of mimicking the construction of the Murrayvon Neumann semigroup in letter rather than in spirit. Each projection in $A \otimes \mathcal{K}$ is equivalent to a projection in some $\mathrm{M}_{n}(A)$, whence $\mathrm{M}_{\infty}(A)$ is large enough to encompass all possible equivalence classes of projections. The same is not true, however, of positive elements and Cuntz equivalence. The definition below amounts essentially to replacing $\mathrm{M}_{\infty}(A)$ with $A \otimes \mathcal{K}$ in the definition above (this is a theorem), and also gives a new and very useful characterisation of Cuntz subequivalence. We refer the reader to [38] and [43] for background material on Hilbert $\mathrm{C}^{*}$-modules.

Consider $A$ as a (right) Hilbert $C^{*}$-module over itself, and let $H_{A}$ denote the countably infinite direct sum of copies of this module. There is a one-to-one correspondence between closed countably generated submodules of $H_{A}$ and hereditary subalgebras of $A \otimes \mathcal{K}$ : the hereditary subalgebra $B$ corresponds to $B H_{A}$. Since $A$ is separable, $B$ is singly hereditarily generated, and it is fairly routine to prove that any two generators are Cuntz equivalent in the sense of Definition 2.3. Thus, passing from positive elements to Cuntz equivalence classes factors through the passage from positive elements to the hereditary subalgebras they generate.

Let $X$ and $Y$ be closed countably generated submodules of $H_{A}$. Recall that the compact operators on $H_{A}$ form a $\mathrm{C}^{*}$-algebra isomorphic to $A \otimes \mathcal{K}$. Let us say that $X$ is compactly contained in $Y$ if $X$ is contained in $Y$ and there is a compact self-adjoint endomorphism of $Y$ which fixes $X$ pointwise. Such an endomorphism extends naturally to a compact self-adjoint endomorphism of $H_{A}$, and so may be viewed as a self-adjoint element of $A \otimes \mathcal{K}$. Let us write $X \precsim Y$ if each closed countably generated compactly contained submodule of $X$ is isomorphic to such a submodule of $Y$.

Theorem 2.4 (Coward-Elliott-Ivanescu, [12]). The relation $\precsim$ on Hilbert $C^{*}$ modules defined above, when viewed as a relation on positive elements in $\mathrm{M}_{\infty}(A)$, is precisely the relation $\precsim$ of Definition 2.3 .

Let $[X]$ denote the Cuntz equivalence class of the module $X$. One may construct a positive ordered Abelian semigroup $\mathcal{C} u(A)$ by endowing the set of countably generated Hilbert $\mathrm{C}^{*}$-modules over $A$ with the operation

$$
[X]+[Y]:=[X \oplus Y]
$$

and the partial order

$$
[X] \leq[Y] \Leftrightarrow X \precsim Y .
$$

The semigroup $\mathcal{C} u(A)$ coincides with $\mathrm{W}(A)$ whenever $A$ is stable, i.e., $A \otimes \mathcal{K} \cong$ $A$, and has some advantages over $\mathrm{W}(A)$ in general. First, suprema of increasing sequences always exist in $\mathcal{C} u(A)$. This leads to the definition of a category including 
this structure in which $\mathcal{C} u(A)$ sits as an object, and as a functor into which it is continuous with respect to inductive limits. (Definition 2.3 casts $\mathrm{W}(A)$ as a functor into just the category of ordered Abelian semigroups with zero. This functor fails to be continuous with respect to inductive limits.) Second, it results in the simplification in the case that $A$ has stable rank one that Cuntz equivalence of positive elements amounts simply to isomorphism of the corresponding Hilbert $\mathrm{C}^{*}$ modules. This has led, via recent work of Brown, Perera, and the second-named author, to the complete classification of all countably generated Hilbert $\mathrm{C}^{*}$-modules over $A$ via $\mathrm{K}_{0}$ and traces, provided that $A$ has the relatively common property of strict comparison (more on this property in Section 3) ([5], [6]), and to the classification of closed unitary orbits of positive operators in $A \otimes \mathcal{K}$ through recent work of Ciuperca and the first-named author ([7]).

Essentially, $\mathrm{W}(A)$ and $\mathcal{C} u(A)$ contain the same information, but we have chosen to maintain separate notation both to avoid confusion and because many results in the literature are stated only for $\mathrm{W}(A)$.

Cuntz equivalence is often described roughly as the Murray-von Neumann equivalence of the support projections of positive elements. This heuristic is, modulo accounting for projections, precise in $\mathrm{C}^{*}$-algebras for which the Elliott invariant is known to be complete ([47]). In the stably finite case, one recovers $\mathrm{K}_{0}$, the tracial simplex, and the pairing $\rho$ (see (1)) from the Cuntz semigroup, whence the invariant

$$
\left(\mathcal{C} u(A), \mathrm{K}_{1} A\right)
$$

is finer than $\operatorname{Ell}(A)$ in general. Remarkably, these two invariants determine each other in a natural way for the largest class of unital stably finite $\mathrm{C}^{*}$-algebras in which (EC) can be expected to hold ([5], [6]). The class in question consists of those algebras which satisfy a certain regularity property, $\mathcal{Z}$-stability, which we shall introduce presently.

\section{Regularity PROPERTIES}

Let us now describe three agreeable properties which a $\mathrm{C}^{*}$-algebra may enjoy. We will see later that virtually all classification theorems for separable amenable $\mathrm{C}^{*}$-algebras via the Elliott invariant assume, either explicitly or implicitly, one of these properties.

3.1. Strict comparison. Our first regularity property - strict comparison - is one that guarantees, in simple $\mathrm{C}^{*}$-algebras, that the heuristic view of Cuntz equivalence described at the end of Section 2 is in fact accurate for positive elements which are not Cuntz equivalent to projections (see [47]). The property is K-theoretic in character.

Let $A$ be a unital $\mathrm{C}^{*}$-algebra, and denote by $\mathrm{QT}(A)$ the space of normalised 2quasitraces on $A$ (v. [2, Definition II.1.1]). Let $\mathrm{S}(\mathrm{W}(A))$ denote the set of additive and order preserving maps $d$ from $\mathrm{W}(A)$ to $\mathbb{R}^{+}$having the property that $d\left(\left\langle 1_{A}\right\rangle\right)=$ 1. Such maps are called states. Given $\tau \in \mathrm{QT}(A)$, one may define a map $d_{\tau}$ : $\mathrm{M}_{\infty}(A)_{+} \rightarrow \mathbb{R}^{+}$by

$$
d_{\tau}(a)=\lim _{n \rightarrow \infty} \tau\left(a^{1 / n}\right) .
$$

This map is lower semicontinuous, and depends only on the Cuntz equivalence class of $a$. It moreover has the following properties:

(i) if $a \precsim b$, then $d_{\tau}(a) \leq d_{\tau}(b)$; 
(ii) if $a$ and $b$ are orthogonal, then $d_{\tau}(a+b)=d_{\tau}(a)+d_{\tau}(b)$.

Thus, $d_{\tau}$ defines a state on $\mathrm{W}(A)$. Such states are called lower semicontinuous dimension functions, and the set of them is denoted by $\operatorname{LDF}(A)$. If $A$ has the property that $a \precsim b$ whenever $d(a)<d(b)$ for every $d \in \operatorname{LDF}(A)$, then let us say that $A$ has strict comparison of positive elements or simply strict comparison.

A theorem of Haagerup asserts that every element of $\mathrm{QT}(A)$ is in fact a trace if $A$ is exact ([31]). All amenable $\mathrm{C}^{*}$-algebras are exact, so we dispense with the consideration of quasi-traces from here on.

3.2. Finite decomposition rank. Our second regularity property, introduced by Kirchberg and Winter, is topological in flavour. It is based on a noncommutative version of covering dimension called decomposition rank.

Definition 3.1 ([37], Definitions 2.2 and 3.1). Let $A$ be a separable $C^{*}$-algebra.

(i) We shall say that a completely positive map $\varphi: \bigoplus_{i=1}^{s} M_{r_{i}} \rightarrow A$ is $n$ decomposable if there is a decomposition $\{1, \ldots, s\}=\coprod_{j=0}^{n} I_{j}$ such that the restriction of $\varphi$ to $\bigoplus_{i \in I_{j}} M_{r_{i}}$ preserves orthogonality for each $j \in\{0, \ldots, n\}$.

(ii) $A$ will be said to have decomposition $\operatorname{rank} n$, denoted by $\operatorname{dr} A=n$, if $n$ is the least integer such that the following holds: Given $\left\{b_{1}, \ldots, b_{m}\right\} \subset A$ and $\epsilon>0$, there is a completely positive approximation $(F, \psi, \varphi)$ for $b_{1}, \ldots, b_{m}$ within $\epsilon$ (i.e., $\psi: A \rightarrow F$ and $\varphi: F \rightarrow A$ are completely positive contractions and $\left.\left\|\varphi \psi\left(b_{i}\right)-b_{i}\right\|<\epsilon\right)$ such that $\varphi$ is $n$-decomposable. If no such $n$ exists, we write $\operatorname{dr} A=\infty$.

Decomposition rank has good permanence properties. It behaves well with respect to quotients, inductive limits, hereditary subalgebras, unitization and stabilization. Its topological flavour comes from the fact that it generalises covering dimension in the commutative case: if $X$ is a locally compact second countable space, then $\operatorname{dr} \mathrm{C}_{0}(X)=\operatorname{dim} X$. We refer the reader to [37] for details.

The regularity property that we are interested in is finite decomposition rank, expressed by the inequality $\mathrm{dr}<\infty$. This can only occur in a stably finite $\mathrm{C}^{*}-$ algebra.

3.3. $\mathcal{Z}$-stability. The Jiang-Su algebra $\mathcal{Z}$ is a simple separable amenable and infinite-dimensional $\mathrm{C}^{*}$-algebra with the same Elliott invariant as $\mathbb{C}([32])$. We say that a second algebra $A$ is $\mathcal{Z}$-stable if $A \otimes \mathcal{Z} \cong A$. $\mathcal{Z}$-stability is our third regularity property. It is very robust with respect to common constructions (see [61]).

The next theorem shows $\mathcal{Z}$-stability to be highly relevant to the classification program. Recall that a pre-ordered Abelian group $\left(G, G^{+}\right)$is said to be weakly unperforated if $n x \in G^{+} \backslash\{0\}$ implies $x \in G^{+}$for any $x \in G$ and $n \in \mathbb{N}$.

Theorem 3.2 (Gong-Jiang-Su, [28]). Let $A$ be a simple unital $C^{*}$-algebra with weakly unperforated $\mathrm{K}_{0}$-group. It follows that

$$
\operatorname{Ell}(A) \cong \operatorname{Ell}(A \otimes \mathcal{Z}) .
$$

Thus, in the setting of weakly unperforated $\mathrm{K}_{0}$, the completeness of $\operatorname{Ell}(\bullet)$ in the simple unital case of the classification program would imply $\mathcal{Z}$-stability. Remarkably, there exist algebras satisfying the hypotheses of the above theorem which are not $\mathcal{Z}$-stable ([51], [56], [57]). 
3.4. Relationships. In general, no two of the regularity properties above are equivalent. The most important general result connecting them is the following theorem of M. Rørdam ([52]):

Theorem 3.3. Let $A$ be a simple, unital, exact, finite, and $\mathcal{Z}$-stable $C^{*}$-algebra. Then, A has strict comparison of positive elements.

We shall see later that for a substantial class of simple, separable, amenable, and stably finite $\mathrm{C}^{*}$-algebras, all three of our regularity properties are equivalent. Moreover, the algebras in this class which do satisfy these three properties also satisfy (EC). There is good reason to believe that the equivalence of these three properties will hold in much greater generality, at least in the stably finite case; in the general case, strict comparison and $\mathcal{Z}$-stability may well prove to be equivalent characterisations of those simple, unital, separable, and amenable $\mathrm{C}^{*}$-algebras which satisfy (EC).

\section{A BRIEF HISTORY}

We will now take a short tour of the classification program's biggest successes, and also the fascinating algebras of Villadsen. We have two goals in mind: to edify the reader unfamiliar with the classification program and to demonstrate that the regularity properties of Section 3 pervade the known confirmations of (EC). This is a new point of view, for when these results were originally proved, there was no reason to think that anything more than simplicity, separability, and amenability would be required to complete the classification program.

We have divided our review of known classification results into three broad categories according to the types of algebras covered: purely infinite algebras, and two formally different types of stably finite algebras. It is beyond the scope of this article to provide an exhaustive list of known classification results, much less demonstrate their connections to our regularity properties. We will thus choose, from each of the three categories above, the classification theorem with the broadest scope, and indicate how the algebras it covers satisfy at least one of our regularity properties.

4.1. Purely infinite simple algebras. We first consider a case where the theory is summarised with one beautiful result. Recall that a simple separable amenable $\mathrm{C}^{*}$-algebra is purely infinite if every non-zero hereditary subalgebra contains an infinite projection (a projection is infinite if it is equivalent, in the sense of Murray and von Neumann, to a proper subprojection of itself; otherwise the projection is finite).

Theorem 4.1 (Kirchberg-Phillips, 1995, [34] and [48]). Let $A$ and $B$ be separable amenable purely infinite simple $C^{*}$-algebras which satisfy the Universal Coefficient Theorem. If there is an isomorphism

$$
\phi: \operatorname{Ell}(A) \rightarrow \operatorname{Ell}(B),
$$

then there is a*-isomorphism $\Phi: A \rightarrow B$ which induces $\phi$.

In the theorem above, the Elliott invariant is somewhat simplified. The hypotheses on $A$ and $B$ guarantee that they are traceless, and that the order structure on $\mathrm{K}_{0}$ is irrelevant. Thus, the invariant is simply the graded group $\mathrm{K}_{0} \oplus \mathrm{K}_{1}$, along with the $\mathrm{K}_{0}$-class of the unit if it exists. The assumption of the Universal Coefficient Theorem (UCT) is required in order to deduce the theorem from a result which is 
formally more general: $A$ and $B$ as in the theorem are $*$-isomorphic if and only if they are KK-equivalent. The question of whether every amenable $\mathrm{C}^{*}$-algebra satisfies the UCT is open.

Which of our three regularity properties are present here? As noted earlier, finite decomposition rank is out of the question. The algebras we are considering are traceless, and so the definition of strict comparison reduces to the following statement: for any two non-zero positive elements $a, b \in A$, we have $a \precsim b$. This, in turn, is often taken as the very definition of pure infiniteness, and can be shown to be equivalent to the definition preceding Theorem 4.1 without much difficulty. Strict comparison is thus satisfied in a slightly vacuous way. As it turns out, $A$ and $B$ are also $\mathcal{Z}$-stable, although this is less obvious. One first proves that $A$ and $B$ are approximately divisible (again, this does not require Theorem 4.1) and then uses the fact, due to Winter and the second-named author, that any separable and approximately divisible $\mathrm{C}^{*}$-algebra is $\mathcal{Z}$-stable ([62]).

4.2. The stably finite case, I: inductive limits. We now move on to the case of stably finite $\mathrm{C}^{*}$-algebras, i.e., those algebras $A$ such that that every projection in the (unitization of) each matrix algebra $\mathrm{M}_{n}(A)$ is finite. (The question of whether a simple amenable $\mathrm{C}^{*}$-algebra must always be purely infinite or stably finite was recently settled negatively by Rørdam. We will address his example again later.) Many of the classification results in this setting apply to classes of $\mathrm{C}^{*}$-algebras which can be realised as inductive limits of certain building block algebras. The original classification result for stably finite algebras is due to Glimm. Recall that a $\mathrm{C}^{*}$-algebra $A$ is uniformly hyperfinite (UHF) if it is the limit of an inductive sequence

$$
\mathrm{M}_{n_{1}} \stackrel{\phi_{1}}{\longrightarrow} \mathrm{M}_{n_{2}} \stackrel{\phi_{2}}{\longrightarrow} \mathrm{M}_{n_{3}} \stackrel{\phi_{3}}{\longrightarrow} \cdots,
$$

where each $\phi_{i}$ is a unital $*$-homomorphism. We will state his result here as a confirmation of the Elliott conjecture, but note that it predates both the classification program and the realisation that K-theory is the essential invariant.

Theorem 4.2 (Glimm, 1960, [25]). Let $A$ and $B$ be UHF algebras, and suppose that there is an isomorphsim

$$
\phi: \operatorname{Ell}(A) \rightarrow \operatorname{Ell}(B) .
$$

It follows that there is a *-isomorphism $\Phi: A \rightarrow B$ which induces $\phi$.

Again, the invariant is dramatically simplified here. Only the ordered $\mathrm{K}_{0}$-group is non-trivial. The strategy of Glimm's proof (which did not use K-theory explicitly) was to "intertwine" two inductive sequences $\left(\mathrm{M}_{n_{i}}, \phi_{i}\right)$ and $\left(\mathrm{M}_{m_{i}}, \psi_{i}\right)$, i.e., to find sequences of $*$-homomorphisms $\eta_{i}$ and $\gamma_{i}$ making the diagram

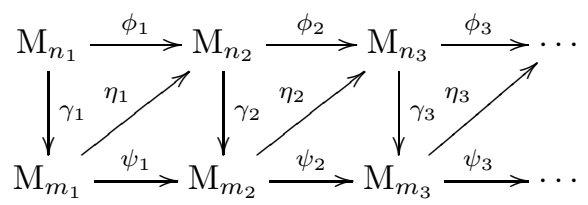

commute. One then gets an isomorphism between the limit algebras by extending the obvious morphism between the inductive sequences by continuity.

The intertwining argument above can be pushed surprisingly far. One replaces the inductive sequences above with more general inductive sequences $\left(A_{i}, \phi_{i}\right)$ and 
$\left(B_{i}, \psi_{i}\right)$, where the $A_{i}$ and $B_{i}$ are drawn from a specified class (matrix algebras over circles, for instance), and seeks maps $\eta_{i}$ and $\gamma_{i}$ as before. Usually, it is not possible to find $\eta_{i}$ and $\gamma_{i}$ making the diagram commute, but approximate commutativity on ever larger finite sets can be arranged, and this suffices for the existence of an isomorphism between the limit algebras. This generalised intertwining is known as the Elliott Intertwining Argument.

The most important classification theorem for inductive limits covers the socalled approximately homogeneous $(\mathrm{AH})$ algebras. An $\mathrm{AH}$ algebra $A$ is the limit of an inductive sequence $\left(A_{i}, \phi_{i}\right)$, where each $A_{i}$ is semi-homogeneous:

$$
A_{i}=\bigoplus_{j=1}^{n_{i}} p_{i, j}\left(\mathrm{C}\left(X_{i, j}\right) \otimes \mathcal{K}\right) p_{i, j}
$$

for some natural number $n_{i}$, compact metric spaces $X_{i, j}$, and projections $p_{i, j} \in$ $\mathrm{C}\left(X_{i, j}\right) \otimes \mathcal{K}$. We refer to the sequence $\left(A_{i}, \phi_{i}\right)$ as a decomposition for $A$; such decompositions are not unique. All AH algebras are separable and amenable.

Let $A$ be a simple unital AH algebra. Let us say that $A$ has slow dimension growth if it has a decomposition $\left(A_{i}, \phi_{i}\right)$ satisfying

$$
\limsup _{i \rightarrow \infty} \sup \left\{\frac{\operatorname{dim}\left(X_{i, 1}\right)}{\operatorname{rank}\left(p_{i, 1}\right)}, \ldots, \frac{\operatorname{dim}\left(X_{i, n_{i}}\right)}{\operatorname{rank}\left(p_{i, n_{i}}\right)}\right\}=0 .
$$

Let us say that $A$ has very slow dimension growth if it has a decomposition satisfying the (formally) stronger condition that

$$
\limsup _{i \rightarrow \infty} \sup \left\{\frac{\operatorname{dim}\left(X_{i, 1}\right)^{3}}{\operatorname{rank}\left(p_{i, 1}\right)}, \ldots, \frac{\operatorname{dim}\left(X_{i, n_{i}}\right)^{3}}{\operatorname{rank}\left(p_{i, n_{i}}\right)}\right\}=0 .
$$

Finally, let us say that $A$ has bounded dimension if there is a constant $M>0$ and a decomposition of $A$ satisfying

$$
\sup _{i, l}\left\{\operatorname{dim}\left(X_{i, l}\right)\right\} \leq M .
$$

Theorem 4.3 (Elliott-Gong, Dădărlat, and Gong, [21], [14] and [27]). (EC) holds among simple unital AH algebras with slow dimension growth and real rank zero.

Theorem 4.4 (Elliott-Gong-Li and Gong, [23] and [26]). (EC) holds among simple unital AH algebras with very slow dimension growth.

All three of our regularity properties hold for the algebras of Theorems 4.3 and 4.4, but some are easier to establish than others. Let us first point out that an algebra from either class has stable rank one and weakly unperforated $\mathrm{K}_{0}$-group (cf. [1]), and that these facts predate Theorems 4.3 and 4.4. A simple unital $\mathrm{C}^{*}$ algebra of real rank zero and stable rank one has strict comparison if and only if its $\mathrm{K}_{0}$-group is weakly unperforated (cf. [46]), whence strict comparison holds for the algebras covered by Theorem 4.3. A recent result of the second-named author shows that strict comparison holds for any simple unital AH algebra with slow dimension growth $([60])$, and this result is independent of the classification theorems above. Thus, strict comparison holds for the algebras of Theorems 4.3 and 4.4, and the proof of this fact, while not easy, is at least much less complicated than the proofs of the classification theorems themselves. Establishing finite decomposition rank requires the full force of the classification theorems: a consequence of both theorems is that the algebras they cover are all in fact simple unital $\mathrm{AH}$ algebras of bounded 
dimension, and such algebras have finite decomposition rank by [37, Corollary 3.12 and 3.3 (ii)]. Proving $\mathcal{Z}$-stability is also an application of Theorems 4.3 and 4.4: one may use these theorems to prove that the algebras in question are approximately divisible $([22])$, and this entails $\mathcal{Z}$-stability for separable $\mathrm{C}^{*}$-algebras ([62]).

Why all the interest in inductive limits? Initially at least, it was surprising to find that any classification of $\mathrm{C}^{*}$-algebras by $\mathrm{K}$-theory was possible, and the earliest theorems to this effect considered inductive limits (see, for instance, the first-named author's classification of AF algebras and AT-algebras of real rank zero in [16] and [18], respectively; it should be pointed out that [16] is based not only on [25] but on the generalisation of Glimm's approach to the full class of AF algebras by Bratteli in [3] - in which even the class of AF algebras is mentioned for the first time). But it was the realisation by Evans and the first-named author that a very natural class of $\mathrm{C}^{*}$-algebras arising from dynamical systems - the irrational rotation algebraswere in fact inductive limits of elementary building blocks that began the drive to classify inductive limits of all stripes ([20]). This theorem of Elliott and Evans has recently been generalised in sweeping fashion by Lin and Phillips, who prove that virtually every $\mathrm{C}^{*}$-dynamical system giving rise to a simple algebra is an inductive limit of fairly tractable building blocks ([42]). This result continues to provide strong motivation for the study of inductive limit algebras.

4.3. The stably finite case, II: tracial approximation. Natural examples of separable amenable $\mathrm{C}^{*}$-algebras are rarely equipped with obvious and useful inductive limit decompositions. Even the aforementioned theorem of Lin and Phillips, which gives an inductive limit decomposition for each minimal $\mathrm{C}^{*}$-dynamical system, does not produce inductive sequences covered by existing classification theorems. It is thus desirable to have theorems confirming the Elliott conjecture under hypotheses that are (reasonably) straightforward to verify for algebras not given as inductive limits.

Lin in [39] introduced the concept of tracial topological rank for $\mathrm{C}^{*}$-algebras. His definition, in spirit if not in letter, is this: a unital simple tracial $\mathrm{C}^{*}$-algebra $A$ has tracial topological rank at most $n \in \mathbb{N}$ if for any finite set $\mathcal{F} \subseteq A$, tolerance $\epsilon>0$, and positive element $a \in A$ there exist unital subalgebras $B$ and $C$ of $A$ such that

(i) $\mathbf{1}_{A}=\mathbf{1}_{B} \oplus \mathbf{1}_{C}$,

(ii) $\mathcal{F}$ is almost (to within $\epsilon$ ) contained in $B \oplus C$,

(iii) $C$ is isomorphic to $F \otimes \mathrm{C}(X)$, where $\operatorname{dim}(X) \leq n$ and $F$ is finite-dimensional, and

(iv) $\mathbf{1}_{B}$ is dominated, in the sense of Cuntz subequivalence, by $a$.

One denotes by $\operatorname{TR}(A)$ the least integer $n$ for which $A$ satisfies the definition above; this is the tracial topological rank, or simply the tracial rank, of $A$.

The most important value of the tracial rank is zero. Lin proved that simple unital separable amenable $\mathrm{C}^{*}$-algebras of tracial rank zero satisfy the Elliott conjecture, modulo the ever present UCT assumption ([40]). The great advantage of this result is that its hypotheses can be verified for a wide variety of $\mathrm{C}^{*}$-dynamical systems and all simple non-commutative tori, without ever having to prove that the latter have tractable inductive limit decompositions (see [49], for instance). Indeed, the existence of such decompositions is a consequence of Lin's theorem! (Rather, it is a consequence of his proof, which showed that his class coincided with that of 
[21].) One can also verify the hypotheses of Lin's classification theorem for many real rank zero $\mathrm{C}^{*}$-algebras with unique trace $([4])$, always with the assumption, indirectly, of strict comparison.

Simple unital $\mathrm{C}^{*}$-algebras of tracial rank zero can be shown to have stable rank one and weakly unperforated $\mathrm{K}_{0}$-group, whence they have strict comparison of positive elements by a theorem of Perera ([46]). (There is a classification theorem for algebras of tracial rank one ([41]), but this has been somewhat less useful-it is difficult to verify tracial rank one in natural examples. Also, Niu has recently proved a classification theorem for some $\mathrm{C}^{*}$-algebras which are approximated in trace by certain subalgebras of $\mathrm{M}_{n} \otimes \mathrm{C}[0,1]$ ([44], [45]).)

And what of our regularity properties? Lin proved in [39] that every unital simple $\mathrm{C}^{*}$-algebra of tracial rank zero has stable rank one and weakly unperforated $\mathrm{K}_{0^{-}}$ group. These facts, by the results reviewed at the end of the preceding subsection, entail strict comparison and are not nearly so difficult to prove as the tracial rank zero classification theorem. In a further analogy with the case of AH algebras, finite decomposition rank and $\mathcal{Z}$-stability can only be verified by applying Lin's classification theorem - a consequence of this theorem (or rather, its proof; cf. above) is that the algebras it covers are in fact $\mathrm{AH}$ algebras of bounded dimension!

4.4. Villadsen's algebras. Until the mid 1990s we had no examples of simple separable amenable $\mathrm{C}^{*}$-algebras where one of our regularity properties failed. To be fair, two of our regularity properties had not yet even been defined, and strict comparison was seen as a technical version of the more attractive Second Fundamental Comparability Question for projections (this last condition, abbreviated FCQ2, asks for strict comparison for projections only). This all changed when Villadsen produced a simple separable amenable and stably finite $\mathrm{C}^{*}$-algebra which did not have FCQ2, answering a long-standing question of Blackadar ([64]). The techniques introduced by Villadsen were subsequently used by him and others to answer many open questions in the theory of nuclear $\mathrm{C}^{*}$-algebras including the following:

(i) Does there exist a simple separable amenable $\mathrm{C}^{*}$-algebra containing a finite and an infinite projection? (Solved affirmatively by Rørdam in [51].)

(ii) Does there exist a simple and stably finite $\mathrm{C}^{*}$-algebra with non-minimal stable rank? (Solved affirmatively by Villadsen in [65].)

(iii) Is stability a stable property for simple $\mathrm{C}^{*}$-algebras? (Solved negatively by Rørdam in [53].)

(iv) Does a simple and stably finite $\mathrm{C}^{*}$-algebra with cancellation of projections necessarily have stable rank one? (Solved negatively by the second-named author in [59].)

(v) Are the $\mathrm{C}^{*}$-algebras of minimal dynamical systems always classified by their Elliott invariants? (Solved negatively by Kerr and Giol in [33].)

Of the results above, (i) was (and is) the most significant. In addition to showing that simple separable amenable $\mathrm{C}^{*}$-algebras do not have a factor-like type classification, Rørdam's example demonstrated that the Elliott invariant as it stood could not be complete in the simple case. This and other examples due to the secondnamed author have necessitated a revision of the classification program ([58], [59]). It is to the nature of this revision that we now turn. 


\section{The WAY(s) FORWARD}

5.1. New assumptions. (EC) does not hold in general, and this justifies new assumptions in efforts to confirm it. In particular, one may assume any combination of our three regularity properties. We will comment on the aptness of these new assumptions in the next subsection. For now we observe that, from a certain point of view, we have been making these assumptions all along. Existing classification theorems for stably finite $\mathrm{C}^{*}$-algebras of real rank zero are accompanied by the crucial assumptions of stable rank one and weakly unperforated $\mathrm{K}_{0}$; as has already been pointed out, unperforated $\mathrm{K}_{0}$ can be replaced with strict comparison in this setting.

How much further can one get by assuming the (formally) stronger condition of $\mathcal{Z}$-stability? What role does finite decomposition rank play? As it turns out, these two properties both alone and together produce interesting results. Let $\mathcal{R} \mathcal{R} 0$ denote the class of simple unital separable amenable $\mathrm{C}^{*}$-algebras of real rank zero. The following subclasses of $\mathcal{R} \mathcal{R} 0$ satisfy (EC):

(i) algebras that satisfy the UCT, have finite decomposition rank, and have tracial simplex with compact and zero-dimensional extreme boundary;

(ii) $\mathcal{Z}$-stable algebras that satisfy the UCT and are approximated locally by subalgebras of finite decomposition rank.

These results, due to Winter ([66], [67]), showcase the power of our regularity properties: included in the algebras covered by (ii) are all simple separable unital $\mathcal{Z}$-stable ASH (approximately subhomogeneous) algebras of real rank zero.

Another advantage to the assumptions of $\mathcal{Z}$-stability and strict comparison is that they allow one to recover extremely fine isomorphism invariants for $\mathrm{C}^{*}$-algebras from the Elliott invariant alone. (This recovery is not possible in general.) We will be able to give precise meaning to this comment below, but first require a further discussion of the Cuntz semigroup.

5.2. New invariants. A natural reaction to an incomplete invariant is to enlarge it: include whatever information was used to prove incompleteness. This is not always a good idea. It is possible that one's distinguishing information is ad hoc and unlikely to yield a complete invariant. Worse, one may throw so much new information into the invariant that the impact of its potential completeness is severely diminished. The revision of an invariant is a delicate business. In this light, not all counterexamples are equal.

Rørdam's finite-and-infinite-projection example is distinguished from a simple and purely infinite algebra with the same K-theory by the obvious fact that the latter contains no finite projections. The natural invariant which captures this difference is the semigroup of Murray-von Neumann equivalence classes of projections in matrices over an algebra $A$, denoted by $\mathrm{V}(A)$. After the appearance of Rørdam's example, the second-named author produced a pair of simple, separable, amenable, and stably finite $\mathrm{C}^{*}$-algebras which agreed on the Elliott invariant but were not isomorphic. In this case the distinguishing invariant was Rieffel's stable rank. It was later discovered that these algebras could not be distinguished by their Murrayvon Neumann semigroups, but it was not yet clear which data were missing from the Elliott invariant. More dramatic examples were needed, ones which agreed on most candidates for enlarging the invariant and pointed the way to the "missing information". 
In [57], the second-named author constructed a pair of simple unital AH algebras which, while non-isomorphic, agreed on a wide swath of invariants including the Elliott invariant, all continuous (with respect to inductive sequences) and homotopy invariant functors from the category of $\mathrm{C}^{*}$-algebras (a class which includes the Murray-von Neumann semigroup), the real and stable ranks, and, as was shown later in [58], stable isomorphism invariants (those invariants which are insensitive to tensoring with a matrix algebra or passing to a hereditary subalgebra). It seemed reasonable to expect that the distinguishing invariant in this example - the Cuntz semigroup - might be a good candidate for enlarging the invariant. At least, it was an object which after years of being used sparingly as a means to other ends, merited study for its own sake.

Let us collect some evidence supporting the addition of the Cuntz semigroup to the usual Elliott invariant. First, in the biggest class of algebras where (EC) can be expected to hold- $\mathcal{Z}$-stable algebras, as shown by Theorem 3.2 - it is not an addition at all! Recent work of Brown, Perera, and the second-named author shows that for a simple unital separable amenable $\mathrm{C}^{*}$-algebra which absorbs $\mathcal{Z}$ tensorially, there is a functor which recovers the Cuntz semigroup from the Elliott invariant ([5], [47]). This functorial recovery also holds for simple unital AH algebras of slow dimension growth, a class for which $\mathcal{Z}$-stability is not known and yet confirmation of (EC) is expected. (It should be noted that the computation of the Cuntz semigroup for a simple approximate interval (AI) algebra was essentially carried out by Ivanescu and the first-named author in [24], although one does require [12, Corollary 4] to see that the computation is complete.)

Second, the Cuntz semigroup unifies the counterexamples of Rørdam and the second-named author. One can show that the examples of [50], [56], and [57] all consist of pairs of algebras with different Cuntz semigroups; there are no known counterexamples to the conjecture that simple separable amenable $\mathrm{C}^{*}$-algebras will be classified up to $*$-isomorphism by the Elliott invariant and the Cuntz semigroup.

Third, the Cuntz semigroup provides a bridge to the classification of non-simple algebras. Ciuperca and the first-named author have recently proved that AI algebras - limits of inductive sequences of algebras of the form

$$
\bigoplus_{i=1}^{n} \mathrm{M}_{m_{i}}(\mathrm{C}[0,1])
$$

- are classified up to isomorphism by their Cuntz semigroups ([7]). This is accomplished by proving that the approximate unitary equivalence classes of positive operators in the unitization of a stable $\mathrm{C}^{*}$-algebra of stable rank one are determined by the Cuntz semigroup of the algebra, and then appealing to a theorem of Thomsen $([55])$. (These approximate unitary equivalence classes of positive operators can be endowed with the structure of a metric Abelian semigroup with functional calculus. This invariant, known as Thomsen's semigroup, is recovered functorially in [7] from the Cuntz semigroup for a $\mathrm{C}^{*}$-algebra of stable rank one, and so from the Elliott invariant in an algebra which is moreover simple, unital, exact, finite, and $\mathcal{Z}$-stable by the results of [5]. Thomsen's semigroup is the fine invariant alluded to at the end of Subsection 5.1.)

There is one last reason to suspect a deep connection between the classification program and the Cuntz semigroup. Let us first recall a theorem of Kirchberg, which is germane to the classification of purely infinite $\mathrm{C}^{*}$-algebras (cf. Theorem 4.1). 
Theorem 5.1 (Kirchberg, c. 1994; see [36]). Let $A$ be a separable amenable $C^{*}$ algebra. The following two properties are equivalent:

(i) A is purely infinite;

(ii) $A \otimes \mathcal{O}_{\infty} \cong A$.

A consequence of Kirchberg's theorem is that among simple separable amenable $\mathrm{C}^{*}$-algebras which merely contain an infinite projection, there is a two-fold characterisation of the (proper) subclass which satisfies the original form of the Elliott conjecture (modulo UCT). If one assumes a priori that $A$ is simple and unital with no tracial state, then a theorem of Rørdam (see [52]) shows that the property (ii) above, known as $\mathcal{O}_{\infty}$-stability, is equivalent to $\mathcal{Z}$-stability. Under these same hypotheses, the property (i) is equivalent to the statement that $A$ has strict comparison. Kirchberg's theorem can thus be rephrased as follows in the simple unital case:

Theorem 5.2. Let $A$ be a simple separable unital amenable $C^{*}$-algebra without a tracial state. The following two properties are equivalent:

(i) A has strict comparison;

(ii) $A \otimes \mathcal{Z} \cong A$.

The properties (i) and (ii) in the theorem above make perfect sense in the presence of a trace. We moreover have that (ii) implies (i) even in the presence of traces (this is due to Rørdam; see [52]). It therefore makes sense to ask whether the theorem might be true without the tracelessness hypothesis. Remarkably, this appears to be the case. Winter and the second-named author have proved that for a substantial class of stably finite $\mathrm{C}^{*}$-algebras, strict comparison and $\mathcal{Z}$-stability are equivalent, and that these properties moreover characterise the (proper) subclass which satisfies (EC) ([63]). In other words, Kirchberg's theorem is quite possibly a special case of a more general result, one which will give a unified two-fold characterisation of those simple separable amenable $\mathrm{C}^{*}$-algebras which satisfy the original form of the Elliott conjecture.

It is too soon to know whether the Cuntz semigroup together with the Elliott invariant will suffice for the classification of simple separable amenable $\mathrm{C}^{*}$-algebras, or indeed, whether such a broad classification can be hoped for at all. But there is already cause for optimism. Zhuang Niu has recently obtained some results on lifting maps at the level of the Cuntz semigroup to $*$-homomorphisms. This type of lifting result is a key ingredient in proving almost any kind of classification theorem (cf. [19]). His results suggest the algebras of [57] as the appropriate starting point for any effort to establish the Cuntz semigroup as a complete isomorphism invariant, at least in the absence of $\mathrm{K}_{1}$.

We close our survey with a few questions for the future, both near and far.

(i) When do natural examples of simple separable amenable $\mathrm{C}^{*}$-algebras satisfy one or more of the regularity properties of Section 3? In particular, do simple unital inductive limits of recursive subhomogeneous algebras have strict comparison whenever they have slow dimension growth?

(ii) Can the classification of self-adjoint operators up to approximate unitary equivalence via the Cuntz semigroup in algebras of stable rank one be extended to normal elements, provided that one accounts for $\mathrm{K}_{1}$ ? 
(iii) Let $A$ be a simple, unital, separable, and amenable $\mathrm{C}^{*}$-algebra with strict comparison of positive elements. Is $A \mathcal{Z}$-stable? Less ambitiously, does $A$ have stable rank one whenever it is stably finite?

(iv) Can one use Thomsen's semigroup to prove new classification theorems? (The attraction here is that Thomsen's semigroup is implicit in the Elliott invariant for many $\mathrm{C}^{*}$-algebras. Note that by [7], in the case of stable rank one the Thomsen semigroup contains exactly the same information as the Cuntz semigroup.)

\section{About The AUthors}

George Elliott holds a Canada Research Chair at the University of Toronto. He is a Distinguished Senior Member of the Fields Institute for Research in Mathematical Sciences and an honorary professor at the University of Copenhagen and the University of Wales, Cardiff.

Andrew Toms is an assistant professor at York University. He has held an NSERC Postdoctoral Fellowship at the Fields Institute for Research in Mathematical Sciences and at the University of Copenhagen.

\section{REFERENCES}

[1] Blackadar, B., Dădărlat, M., and Rørdam, M.: The real rank of inductive limit C*-algebras, Math. Scand. 69 (1991), 211-216. MR1156427 (93e:46067)

[2] Blackadar, B., and Handelman, D.: Dimension functions and traces on $C^{*}$-algebras, J. Funct. Anal. 45 (1982), 297-340. MR650185 (83g:46050)

[3] Bratteli, O.: Inductive limits of finite dimensional $C^{*}$-algebras, Trans. Amer. Math. Soc. 171 (1972), 195-234. MR0312282 (47:844)

[4] Brown, N.: Invariant means and finite representation theory of $C^{*}$-algebras, Mem. Amer. Math. Soc. 184 (2006), no. 865, viii+105 pp. MR2263412

[5] Brown, N. P., Perera, F., and Toms, A. S.: The Cuntz semigroup, the Elliott conjecture, and dimension functions on $C^{*}$-algebras, to appear in J. Reine Angew. Math.

[6] Brown, N. P., and Toms, A. S.: Three applications of the Cuntz semigroup, to appear in Int. Math. Res. Not.

[7] Ciuperca, A., and Elliott, G. A.: A remark on invariants for $C^{*}$-algebras of stable rank one, preprint.

[8] Choi, M.-D., and Effros, E. G.: Nuclear $C^{*}$-algebras and the approximation property, Amer. J. Math. 100 (1978), 61-79. MR0482238 (58:2317)

[9] Choi, M.-D., and Effros, E. G.: Nuclear $C^{*}$-algebras and injectivity: the general case, Indiana Univ. Math. J. 26 (1977), 443-446. MR0430794 (55:3799)

[10] Connes, A.: On the cohomology of operator algebras, J. Funct. Anal. 28 (1978), 248-253. MR0493383 (58:12407)

[11] Connes, A.: Classification of injective factors. Cases $I I_{1}, I I_{\infty}, I I I_{\lambda}, \lambda \neq 1$, Ann. of Math. (2) 104 (1976), 73-115. MR0454659 (56:12908)

[12] Coward, K. T., Elliott, G. A., and Ivanescu, C.: The Cuntz semigroup as an invariant for $C^{*}$-algebras, electronic preprint, arXiv:0705.0341 (2007).

[13] Cuntz, J.: Dimension functions on simple $C^{*}$-algebras, Math. Ann. 233 (1978), 145-153. MR0467332 (57:7191)

[14] Dădărlat, M.: Reduction to dimension three of local spectra of real rank zero $C^{*}$-algebras, J. Reine Angew. Math. 460 (1995), 189-212. MR1316577 (95m:46116)

[15] Dădărlat, M.: Nonnuclear subalgebras of AF algebras, Amer. J. Math. 122 (2000), 581-597. MR1759889 (2001g:46141)

[16] Elliott, G. A.: On the classification of inductive limits of sequences of semi-simple finitedimensional algebras, J. Algebra 38 (1976), 29-44. MR0397420 (53:1279)

[17] Elliott, G. A.: The classification problem for amenable $C^{*}$-algebras, Proceedings of ICM '94, Zurich, Switzerland, Birkhäuser Verlag, Basel, Switzerland, 922-932. MR1403992 (97g:46072) 
[18] Elliott, G. A.: On the classification of $C^{*}$-algebras of real rank zero, J. Reine Angew. Math. 443 (1993), 179-219. MR1241132 (94i:46074)

[19] Elliott, G. A.: Towards a theory of classification, to appear in Advances in Math.

[20] Elliott, G. A., and Evans, D. E.: The structure of the irrational rotation $C^{*}$-algebra, Ann. of Math. (2) 138 (1993), 477-501. MR1247990 (94j:46066)

[21] Elliott, G. A., and Gong, G.: On the classification of $C^{*}$-algebras of real rank zero. II. Ann. of Math. (2) 144 (1996), 497-610. MR1426886 (98j:46055)

[22] Elliott, G. A., Gong, G., and Li, L.: Approximate divisibility of simple inductive limit $C^{*}$ algebras, Contemp. Math. 228, Amer. Math. Soc., Providence, RI (1998), 87-97. MR1667656 (2000k:46078)

[23] Elliott, G. A., Gong, G., and Li, L.: On the classification of simple inductive limit $C^{*}$ algebras, II: The isomorphism theorem, Invent. Math. 168 (2007), 249-320. MR2289866

[24] Elliott. G. A., and Ivanescu, C.: The classification of separable simple $C^{*}$-algebras which are inductive limits of continuous-trace $C^{*}$-algebras with spectrum homeomorphic to the closed interval [0,1], J. Funct. Anal. (2008).

[25] Glimm, J.: On a certain class of operator algebras, Trans. Amer. Math. Soc. 95 (1960), 318-340. MR0112057 (22:2915)

[26] Gong, G.: On the classification of simple inductive limit $C^{*}$-algebras $I$. The reduction theorem., Doc. Math. 7 (2002), 255-461. MR2014489 (2007h:46069)

[27] Gong, G.: On inductive limits of matrix algebras over higher-dimensional spaces, II, Math. Scand. 80 (1997), 56-100. MR1466905 (98j:46061)

[28] Gong, G., Jiang, X., and Su, H.: Obstructions to $\mathcal{Z}$-stability for unital simple $C^{*}$-algebras, Canad. Math. Bull. 43 (2000), 418-426. MR1793944 (2001k:46086)

[29] Haagerup, U.: All nuclear $C^{*}$-algebras are amenable, Invent. Math. 74 (1983), 305-319. MR723220 (85g:46074)

[30] Haagerup, U.: Connes' bicentralizer problem and uniqueness of the injective factor of type $I I I_{1}$, Acta. Math. 158 (1987), 95-148. MR880070 (88f:46117)

[31] Haagerup, U.: Quasi-traces on exact $C^{*}$-algebras are traces, preprint (1991).

[32] Jiang, X. and Su, H.: On a simple unital projectionless $C^{*}$-algebra, Amer. J. Math. 121 (1999), 359-413. MR1680321 (2000a:46104)

[33] Kerr, D., and Giol, J.: Subshifts and perforation, preprint (2007).

[34] Kirchberg, E.: The classification of purely infinite $C^{*}$-algebras using Kasparov's theory, in preparation for Fields Inst. Monograph.

[35] Kirchberg, E.: $C^{*}$-nuclearity implies CPAP, Math. Nachr. 76 (1977), 203-212. MR0512362 $(58: 23623)$

[36] Kirchberg, E., and Phillips, N. C.: Embedding of exact $C^{*}$-algebras in the Cuntz algebra $\mathcal{O}_{2}$, J. Reine Angew. Math. 525 (2000), 17-53. MR1780426 (2001d:46086a)

[37] Kirchberg, E., and Winter, W.: Covering dimension and quasidiagonality, Intern. J. Math. 15 (2004), 63-85. MR2039212 (2005a:46148)

[38] Lance, E. C.: Hilbert $C^{*}-$ modules, London Mathematical Society Lecture Note Series 210, Cambridge University Press, 1995. MR1325694 (96k:46100)

[39] Lin, H.: The tracial topological rank of $C^{*}$-algebras, Proc. London Math. Soc. (3) 83 (2001), 199-234. MR1829565 (2002e:46063)

[40] Lin, H.: Classification of simple $C^{*}$-algebras of tracial topological rank zero, Duke Math. J. 125 (2004), 91-119. MR2097358 (2005i:46064)

[41] Lin, H.: Simple nuclear $C^{*}$-algebras of tracial topological rank one, electronic preprint, arXiv:math/0401240 (2004).

[42] Lin, Q., and Phillips, N. C.: Inductive limit decompositions of $C^{*}$-dynamical systems, in preparation.

[43] Manuilov, V. M., and Troitsky, E. V.: Hilbert $C^{*}$-modules, Translations of Mathematical Monographs 226, American Mathematical Society, 2001. MR2125398 (2005m:46099)

[44] Niu, Z.: A classification of certain tracially approximately subhomogeneous $C^{*}$-algebras, Ph.D. thesis, University of Toronto, 2005.

[45] Niu, Z.: On the classification of TAI algebras, C. R. Math. Acad. Sci. Soc. R. Can. 24 (2004), no. 1, 18-24. MR2036910 (2004m:46145)

[46] Perera, F.: The structure of positive elements for $C^{*}$-algebras of real rank zero, Internat. J. Math. 8 (1997), 383-405. MR1454480 (98i:46058) 
[47] Perera, F., and Toms, A. S.: Recasting the Elliott conjecture, Math. Ann. 338 (2007), 669702. MR2317934

[48] Phillips, N. C.: A classification theorem for nuclear purely infinite simple $C^{*}$-algebras, Doc. Math. 5 (2000), 49-114. MR1745197 (2001d:46086b)

[49] Phillips, N. C.: Every simple higher dimensional noncommutative torus is an AT-algebra, arXiv preprint math.OA/0609783 (2006).

[50] Rørdam, M.: Classification of Nuclear $C^{*}$-Algebras, Encyclopaedia of Mathematical Sciences 126, Springer-Verlag, Berlin, Heidelberg, 2002.

[51] Rørdam, M.: A simple $C^{*}$-algebra with a finite and an infinite projection, Acta Math. 191 (2003), 109-142. MR2020420 (2005m:46096)

[52] Rørdam, M.: The stable and the real rank of $\mathcal{Z}$-absorbing $C^{*}$-algebras, Internat. J. Math. 15 (2004), 1065-1084. MR2106263 (2005k:46164)

[53] Rørdam, M.: Stability of $C^{*}$-algebras is not a stable property, Doc. Math. 2 (1997), 375-386. MR1490456 (98i:46060)

[54] Rørdam, M.: On the structure of simple $C^{*}$-algebras tensored with a UHF-algebra, II, J. Funct. Anal. 107 (1992), 255-269. MR1172023 (93f:46094)

[55] Thomsen, K.: Inductive limits of interval algebras: unitary orbits of positive elements, Math. Ann. 293 (1992), 47-63. MR1162672 (93f:46112)

[56] Toms, A. S.: On the independence of K-theory and stable rank for simple $C^{*}$-algebras, J. Reine Angew. Math. 578 (2005), 185-199. MR2113894 (2005k:46189)

[57] Toms, A. S.: On the classification problem for nuclear $C^{*}$-algebras, to appear in Ann. of Math. (2).

[58] Toms, A. S.: An infinite family of non-isomorphic $C^{*}$-algebras with identical K-theory, to appear in Trans. Amer. Math. Soc.

[59] Toms, A. S.: Cancellation does not imply stable rank one, Bull. London Math. Soc. 38 (2006), 1005-1008. MR2285254

[60] Toms, A. S.: Stability in the Cuntz semigroup of a commutative $C^{*}$-algebra, to appear in Proc. London Math. Soc.

[61] Toms, A. S., and Winter, W.: Strongly self-absorbing $C^{*}$-algebras, Trans. Amer. Math. Soc. 359 (2007), 3999-4029. MR2302521

[62] Toms, A. S., and Winter, W.: Z्Z-stable ASH algebras, to appear in Canad. J. Math.

[63] Toms. A. S., and Winter, W.: The Elliott conjecture for Villadsen algebras of the first type, arXiv preprint math.OA/0611059 (2006).

[64] Villadsen, J.: Simple $C^{*}$-algebras with perforation, J. Funct. Anal. 154 (1998), 110-116. MR1616504 (99j:46069)

[65] Villadsen, J.: On the stable rank of simple $C^{*}$-algebras, J. Amer. Math. Soc. 12 (1999), 1091-1102. MR1691013 (2000f:46075)

[66] Winter, W.: On topologically finite-dimensional simple $C^{*}$-algebras, Math. Ann. 332 (2005), 843-878. MR2179780 (2006i:46102)

[67] Winter, W.: Simple $C^{*}$-algebras with locally finite decomposition rank, J. Funct. Anal. 243 (2007), 394-425. MR2289694

Department of Mathematics, University of Toronto, Toronto, Ontario, Canada, $\mathrm{M} 5 \mathrm{~S} 2 \mathrm{E} 4$

E-mail address: elliott@math.toronto.edu

Department of Mathematics and Statistics, York University, 4700 Keele Street,

Toronto, Ontario, Canada, M3J 1P3

E-mail address: atoms@mathstat.yorku.ca 